Check for updates

Cite this: RSC Adv., 2019, 9, 12189

\title{
Synthesis of novel thermotropic liquid crystalline polymers by a reactive extrusion process $\dagger$
}

Received 19th December 2018 Accepted 13th April 2019

DOI: $10.1039 / \mathrm{c} 8 \mathrm{ra10410g}$

rsc.li/rsc-advances

\author{
Kyunghwan Oh, $\$$ Hoyeon Kim $\ddagger$ and Yongsok Seo (D) *
}

The syntheses of new thermotropic liquid crystalline polymers (TLCPS) were carried out via the polyaddition reactions of diepoxy-containing mesogens and a monoamine (1-naphthylamine). Both bulk polymerization and reactive extrusion were tested. The reaction between the two epoxy rings on the mesogen unit and the primary amine produces a thermotropic liquid crystalline polymer in the extruder. The amine group combines with the two epoxy rings in a single step via a polyaddition reaction to produce thermotropic liquid crystalline polymers without the formation of any by-products. Both polymers were found to exhibit nematic mesophase characteristics, which were examined by using polarized optical microscopy. The new thermotropic liquid crystalline polymers obtained with the bulk reaction have high molecular weights, whereas the polymers synthesized by using reactive extrusion have low molar mass due to their short residence times in the extruder. All the synthesized TLCPs were found to exhibit high thermal stability. Their decomposition temperatures were found to be above $350{ }^{\circ} \mathrm{C}$, but their melting temperatures are low (below $250^{\circ} \mathrm{C}$ ). The liquid crystalline structures of the TLCPs were verified by performing 2D X-ray diffraction measurements. Scanning electron micrographs of the drawn polymer fibers show that the orientation of their morphologies lies predominantly along the direction of the fibers. The polymers synthesized with the reactive extrusion process have the same physical properties as those obtained with the bulk polyaddition reaction. This observation demonstrates the feasibility of the mass production of new TLCPs through reactive extrusion.

\section{Introduction}

The outstanding physical properties of thermotropic liquid crystalline polymers (TLCPs) such as their high rigidity, high strength, excellent dimensional stability, high heat and chemical resistance, and exceptional barrier properties mean that they are super-engineering materials with a wide range of applications. ${ }^{1-5}$ These excellent properties are due to their unique structures, which arise from their mesogenic moieties that retain liquid crystalline order in the melt state. As a result, thermotropic liquid crystalline polymers (TLCPs) form a liquid crystalline structure in the melt state. Thus, they can be used in the production of so-called in situ composites with common engineering thermoplastics by using conventional processing equipments. $^{6-16}$ However, most TLCPs have high thermal stability and high melting temperature, which means their processibility is not easy. Some TLCPs degrade before melting because their melting temperatures exceed their decomposition

RIAM, School of Materials Science and Engineering, College of Engineering, Seoul National University, Kwanakro-1, Kwanakgu, Seoul, 151-744, Republic of Korea. E-mail:ysseo@snu.ac.kr

$\dagger$ Electronic supplementary information (ESI) available: Synthesis details of the mesogenic epoxides, the polymerization process and the table of NMR peak positions. See DOI: $10.1039 / \mathrm{c} 8 \mathrm{ra10410g}$

\$ These authors contribute equally. temperatures. The high processing temperatures of such TLCPs means that it is difficult to compound them with other common thermoplastic polymers to prepare in situ composites; such composites require thermoplastic matrices that can withstand the high transition temperatures or processing temperatures needed to convert the TLCP phase into fibril shapes for thermoplastic matrix reinforcement. ${ }^{6-18}$ Most TLCPs are synthesized via condensation polymerizations, which generally result in low molecular weights. Increasing the molecular weight of a TLCP improves, its mechanical properties, but also increases its melting temperature. Therefore, TLCPs with high molecular weights but low melting or processing temperatures are required. TLCPs with low transition or processing temperatures have been sought for the past 20 years. ${ }^{19-22}$ We have previously reported a new and simple process for the preparation of a novel type of thermotropic liquid crystalline polymers via a polyaddition reaction. ${ }^{19}$ This process consists of the reaction between a diepoxide compound with a primary monoamine (aniline) to produce a linear chain connecting the two epoxy groups. This approach can produce high molar mass TLCPs that have a low processing temperature because no by-products are generated that must be removed. By varying the structure of the diepoxy compound, the processing temperature of the resulting TLCP can be controlled. In this study, new TLCPs were synthesized by performing both reactive extrusion processes 
and bulk polymerizations in a flask. To the best of our knowledge, this is the first report of the synthesis of new TLCPs via a reactive extrusion process, which enables TLCP mass production in a single step. Mass production is feasible because these TLCPs are synthesized via a polyaddition process that does not generate any by-products that require removal. The processing temperature of the TLCPs can be optimized by varying the structure of the diepoxy compound. Hence, our approach enables the achievement both goals simultaneously.

\section{Experimental}

Two diepoxides, LCE1 (p-phenylene-di-4-(2,3-epoxypropyloxy) benzoate) and LCE3 ( $p$-phenylene-di-4-(4,5-epoxypentyloxy) benzoate) (Scheme 1), were prepared by using a reported procedure. ${ }^{22}$ All chemicals were purchased from Aldrich-Sigma Korea. The synthesis procedure can be simply recapitulated as follows: A mixture of 3-bromopropene, ethyl-4hydroxybenzoate, $\mathrm{K}_{2} \mathrm{CO}_{3}$, and acetone was refluxed for $24 \mathrm{~h}$. The solids were filtered off and the solvent was evaporated, then diethylether and water were added to the remnant. The diethylether layer was separated and washed three times with a $10 \%$ $\mathrm{NaOH}$ solution. After evaporation of the diethylether, the residue was boiled in an ethanol/water $(1: 2)$ solution containing $\mathrm{KOH}$ until the solution became clear. The $\mathrm{pH}$ was lowered to 2 by adding $2 \mathrm{~N} \mathrm{HCl}$ solution. After filtration and washing with excess water, the solids were recrystallized in ethanol to yield 4-(2-propyloxy) benzoic acid as a white powder. A mixture of 4-(2-propyloxy) benzoic acid and $\mathrm{SOCl}_{2}$ was refluxed for $2 \mathrm{~h}$. After evaporation of excess $\mathrm{SOCl}_{2}$, pyridine and hydroquinone were added. The mixture was reacted in an acidified aqueous solution, then filtered and washed with a 5\% $\mathrm{Na}_{2} \mathrm{CO}_{3}$ aqueous solution and water. After drying, the solid product (LCE1) was recrystallized in ethyl acetate. LCE1 was purified with column chromatography by using $\mathrm{CH}_{2} \mathrm{Cl}_{2}$ as eluent. After evaporating the solvent, the LCE1 product was recrystallized in ethyl acetate with a yield of 55\%. LCE3 was synthesized by using 5-bromopentene instead of 3-bromopropene. The yield of LCE3 was $69 \%$. More details of the synthetic procedure are provided in the ESI. $\dagger$

Poly(diepoxy naphthalene)s (PDENs, Scheme 2) were first prepared in a flask by performing the melt polymerizations of the synthesized monomers (LCE1 and LCE3 have propyl and pentyl epoxy groups respectively) with a primary monoamine (1naphthylamine). The reactive extrusions were carried out at

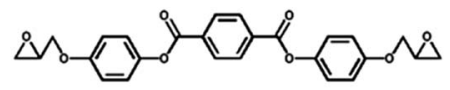

LCE1 (p-phenylene-di[4-(2,3-epoxypropyoxy) benzoate])

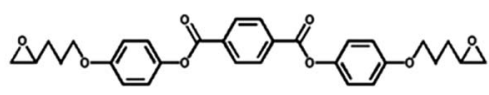

LCE3 (p-phenylene-di[4-(4,5-epoxypentyloxy) benzoate])

Scheme 1 Chemical structures of LCE1 and LCE3.

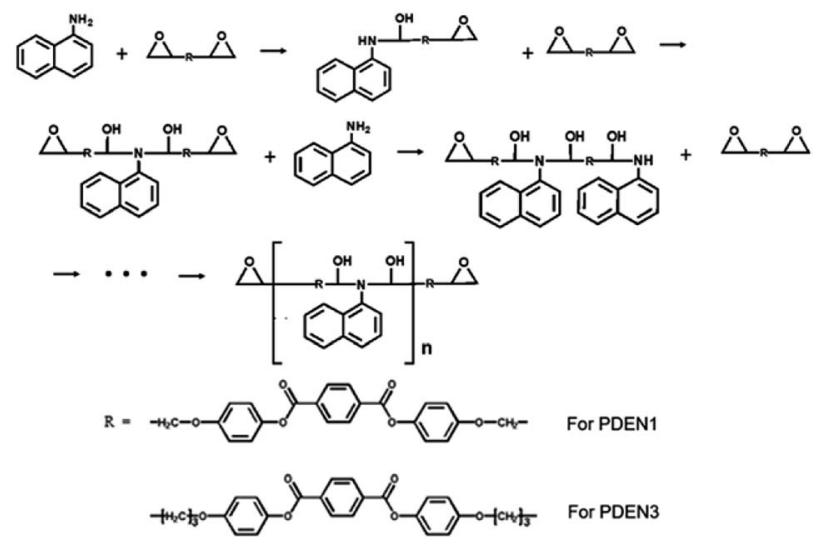

Scheme 2 Synthesis of PDEN1 and PDEN3 via a polyaddition reaction.

a fixed rotation speed of $30 \mathrm{rpm}$ in a $42 \mathrm{~mm}$ Brabender twin screw extruder (AEV651). The extrusion temperatures of the feeding zone/transporting zone/melting zone/die were set as $140 / 220 / 220 / 210{ }^{\circ} \mathrm{C}$, respectively.

Films of the resulting polymers (PDEN1, PDEN1R (PDEN1 synthesized with the reactive extrusion process), PDEN3, and PDEN3R (PDEN3 synthesized with the reactive extrusion process)) were characterized by performing Fourier transform infrared spectrometry (FT-IR, Nicolet 6700, Thermo Scientific (USA)) with an attenuated total reflection (ATR) accessory. The spectra were compiled from 32 scans at a resolution of $8 \mathrm{~cm}^{-1}$. Prior to these measurements, the TLCP samples were hot pressed at $250{ }^{\circ} \mathrm{C}$ and cooled to room temperature to obtain the polymer films.

${ }^{1} \mathrm{H}$-NMR and ${ }^{13} \mathrm{C}$-NMR spectra were obtained with a Bruker spectrometer $(300 \mathrm{MHz})$. The samples were prepared by dissolving the polymers in the solvent THF- $\mathrm{d}_{8}$. The ${ }^{1} \mathrm{H}-\mathrm{NMR}$ spectra were referenced to residual TMS (0 ppm) except in the case of $\mathrm{D}_{2} \mathrm{O}$ (solvent reference, $4.79 \mathrm{ppm}$ ) and DMF- $\mathrm{d}_{7}$ (solvent reference, $8.03 \mathrm{ppm})$. The chemical shifts of the ${ }^{13} \mathrm{C}-\mathrm{NMR}$ spectra were measured relative to DMF- $\mathrm{d}_{7}$ (67.21 and 25.31 ppm).

The thermal properties of the TLCPs, namely their degradation temperatures and transition behaviors, were analyzed by using thermogravimetric analysis (TGA, TGA/DSC1, Mettler Toledo) and differential scanning calorimetry (DSC, DSC 823e, Mettler Toledo). Under a nitrogen atmosphere, the samples were heated in the TGA measurements from 25 to $450{ }^{\circ} \mathrm{C}$ at $10{ }^{\circ} \mathrm{C} \mathrm{min}^{-1}$. In the DSC measurements, each sample was first heated from 25 to $320{ }^{\circ} \mathrm{C}$ at $10{ }^{\circ} \mathrm{C} \mathrm{min}{ }^{-1}$, cooled to $25{ }^{\circ} \mathrm{C}$, and then heated to $320{ }^{\circ} \mathrm{C}$ again at the same heating rate under a nitrogen atmosphere.

Gel permeation chromatography (GPC, Viscotek model 250) was performed with an RI750F refractive index detector. Waters Styragel HP 4E and Styragel HR 5E columns were used with THF as the eluent at a flow rate of $1 \mathrm{~mL} \mathrm{~min}{ }^{-1}$ at $40{ }^{\circ} \mathrm{C}$. Polystyrene standards were used for column calibration.

Polarized light optical microscopy (POM, BX51, Olympus) was used in the polarization mode to observe the thermal transition behaviors of the polymers. The specimens were 
prepared by hot pressing the samples between two slide glasses at $250{ }^{\circ} \mathrm{C}$. The measurement temperature was controlled in the range 25 to $320^{\circ} \mathrm{C}$ with a Mettler Toledo FP82HT hot-stage. The polymers were extruded by using a mini-extruder (DACA 5000) with a pulling unit. Each specimen's morphology was examined with scanning electron microscopy (SEM, Hitachi S 2200C). The surface of each sample fractured in liquid nitrogen was coated with Pt/Pd before the SEM examination.

2-D high temperature X-ray diffraction (XRD) measurements were carried out with a D8 Discover (Bruker) by using the General Area Detector Diffraction System (GADDS) program. $\mathrm{XRD}$ scans were performed with $\mathrm{CuK} \alpha$ radiation from 25 to $230{ }^{\circ} \mathrm{C}$.

\section{Results and discussion}

Fig. 1 shows the FT-IR spectra of the polymers. The sharp absorption peak at $914 \mathrm{~cm}^{-1}$ is due to the vibration of the oxirane ring in LCE1. The reaction between the amine group of 1-naphthyl amine and the oxirane ring (Scheme 2) results in the appearance in the TLCP spectra of a broad shoulder absorption peak at $3450 \mathrm{~cm}^{-1}$, which is ascribed to the stretching vibrations of the produced $\mathrm{OH}$ groups. The intensity of the peak due to the oxirane ring in the TLCPs is significantly reduced. ${ }^{16}$ The epoxide groups on LCE1 and LCE3 react first with the amine group of 1-naphthylamine and then next with the resulting secondary amine to form linear chains (PDEN1 and PDEN3 in Scheme 2). These steps can be repeated alternately to generate high molecular weight polymers.

The ${ }^{1} \mathrm{H}$ NMR spectra of the polymers (PDEN1, PDEN3, PDEN1R, and PDEN3R) were analyzed by using Chemsketch software. The assignment of the signals to the corresponding protons was performed to determine the polymer structures. The mesogenic monomer LCE1 contains five protons on its phenyl rings and three protons on its two aliphatic groups, including those on the methyl groups of the spacer unit and the oxirane ring. All the protons were uniquely identified (Fig. 2(a)). The spectra of PDEN1 and PDEN1R are identical, which

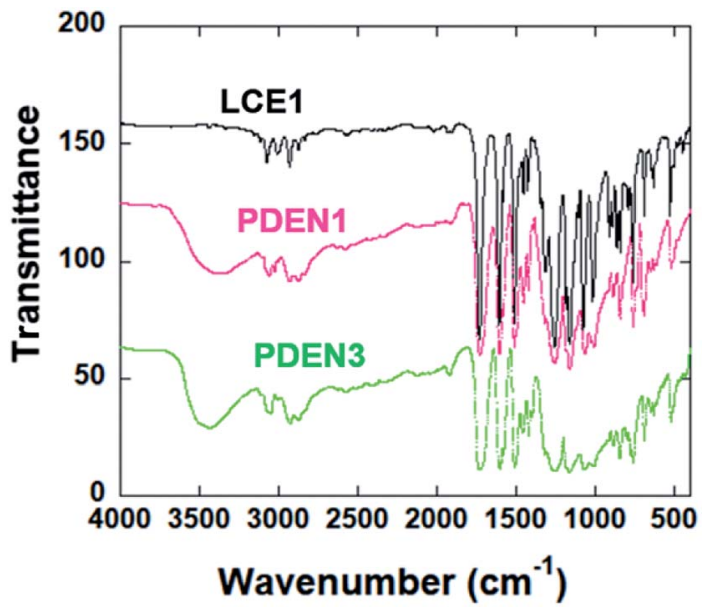

Fig. 1 FTIR of the LCE1, PDEN1 and PDEN3
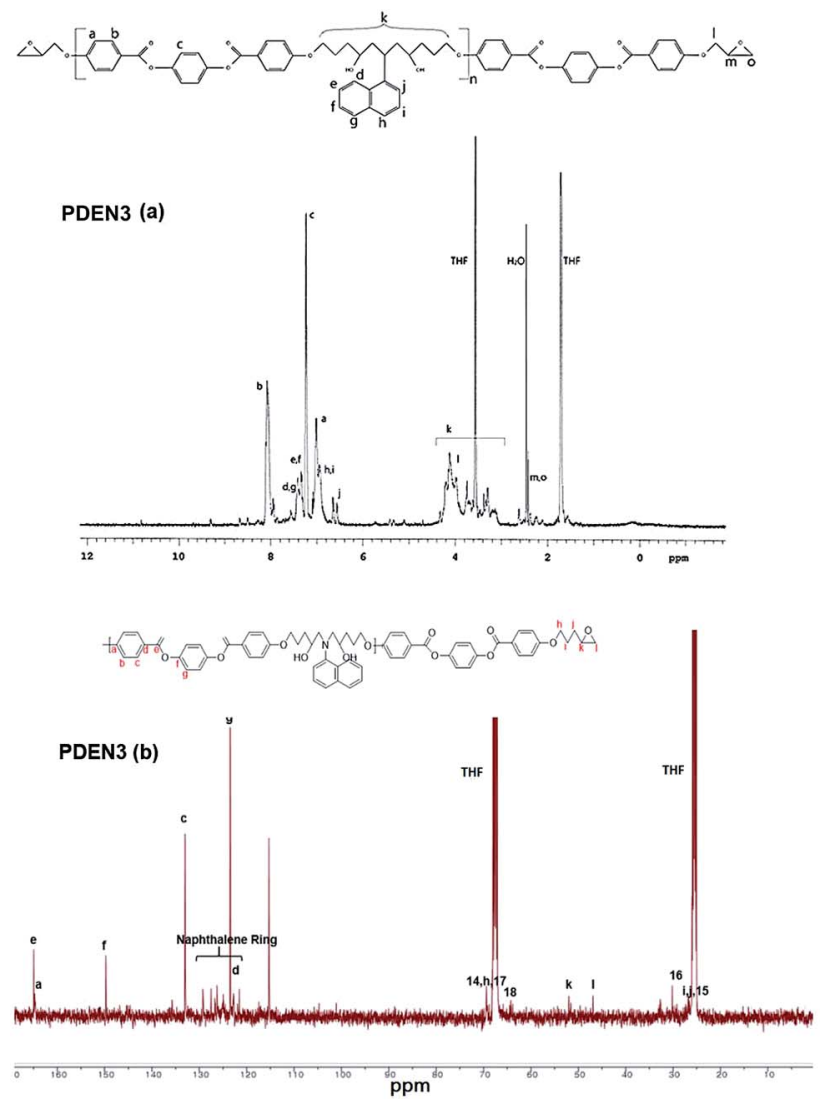

Fig. 2 (a) ${ }^{1} \mathrm{H}$ NMR of PDEN3, (b) ${ }^{13} \mathrm{C}$ NMR of PDEN3.

confirms that they have the same structure (ESI Fig. $3 a$ and $b) . \dagger$ The aromatic proton signals arise at 6.5-8.2 ppm, and the methyl group signals of the TLCPs appear at 3.2-5.3 ppm. The corresponding protons of the LCEs appear at 2.5 and $4.1 \mathrm{ppm}$ respectively. ${ }^{16}$ The reaction between the primary amine group and the epoxy ring proceeds through a ring-opening reaction via nucleophilic attack on the amine, which is accompanied by the appearance of new peaks at 4.7 and $5.3 \mathrm{ppm}$. The doublet at $3.5 \mathrm{ppm}$ is due to the hydrogen atoms in 1-naphthylamine and disappears as the reaction proceeds.

The positions of the peaks in the ${ }^{13} \mathrm{C}$ NMR spectrum of PDEN1 are listed in ESI Fig. 3 and Table $1 . \dagger$ These characteristic peaks are consistent with those of the polymer structures. The characteristic aromatic naphthalene peaks appear at 150.1 and 150.5 ppm, which confirms the formation of PDEN1. These spectral results clearly confirm the polymer structures in Scheme 2. As mentioned above, the first epoxy group reacts vigorously with the amine group to form a compound containing a secondary amine that further reacts with another epoxy group to form a linear polymer chains (Scheme 2). Although the reactivity of the secondary amine group is known to be lower than that of the primary amine groups, it reacts rapidly to form the polymers. ${ }^{\mathbf{2 1 , 2 2}}$

The number and weight average molar masses obtained by using gel permeation chromatography are listed in Table 1 . The weight average molar masses of the bulk synthesized polymers (PDEN1 and PDEN3) are relatively high, although the 
Table 1 Molar mass and the thermal properties of the polymers ${ }^{a}$

\begin{tabular}{|c|c|c|c|c|c|c|}
\hline Samples & $M_{\mathrm{n}}$ & $M_{\mathrm{w}}$ & PDI & $T_{\mathrm{g}}\left({ }^{\circ} \mathrm{C}\right)$ & $T_{\mathrm{m}}\left({ }^{\circ} \mathrm{C}\right)$ & $T_{\mathrm{dg}}\left(5 \mathrm{wt} \%{ }^{\circ} \mathrm{C}\right)$ \\
\hline PDEN1 & 16200 & 32400 & 2 & 100 & 175 & 357 \\
\hline PDEN1R & 10400 & 18200 & 1.8 & 95 & 182 & 352 \\
\hline PDEN3 & 14900 & 46500 & 3.2 & 71 & 193 & 358 \\
\hline PDEN3R & 8600 & 13600 & 1.6 & 73 & 194 & 358 \\
\hline
\end{tabular}

${ }^{a} T_{\mathrm{g}}$ : glass transition temperature, $T_{\mathrm{m}}$ : melt temperature, $T_{\mathrm{dg}}: 5 \mathrm{wt} \%$ degradation temperature.

polydispersity index (PDI) values of PDEN1 and PDEN3 are 2.0 and 3.2, respectively. PDEN3 has a lower molar mass than PDEN1 because the diffusivity of the LCE3 monomer is lower than that of the LCE1 monomer, as found in our previous study. ${ }^{19}$ These large PDI values indicate that the molar mass distribution of the TLCPs are quite broad. In bulk polymerizations, the viscosity increases as the polymerization progresses, which interferes with the diffusion of the LCEs. The polymers synthesized with the reactive extrusion process exhibit better mixing, but the short residence time in the extruder does not allow sufficient reaction time for the preparation of high molar mass polymers. However, the reactive extrusion of LCE1 does result in polymers with a higher molar mass than those of LCE3. The low boiling point of aniline $\left(184^{\circ} \mathrm{C}\right)$ means that it cannot be used in reactive extrusion processes whereas 1-naphthylamine (b.p. $301{ }^{\circ} \mathrm{C}$ ) can be. PDEN1 and PDEN3 have higher molar masses than the other TLCPs since they were prepared via polyaddition reactions without any by-products.

The crystalline structures of the synthesized polymers were investigated with 2-D wide angle X-ray diffraction; the resulting patterns are shown in Fig. 3 and contain inner layer reflections on the meridional scan at a temperature below $T_{\mathrm{m}}$. As for PDEA1, which was synthesized via the reaction between LCE1 and aniline in our previous study, a sharp inner peak is evident at a diffraction angle of $2 \theta=7^{\circ}$, which corresponds to a packing distance of $11.7 \AA$ according to Bragg's law. Wider outer peaks
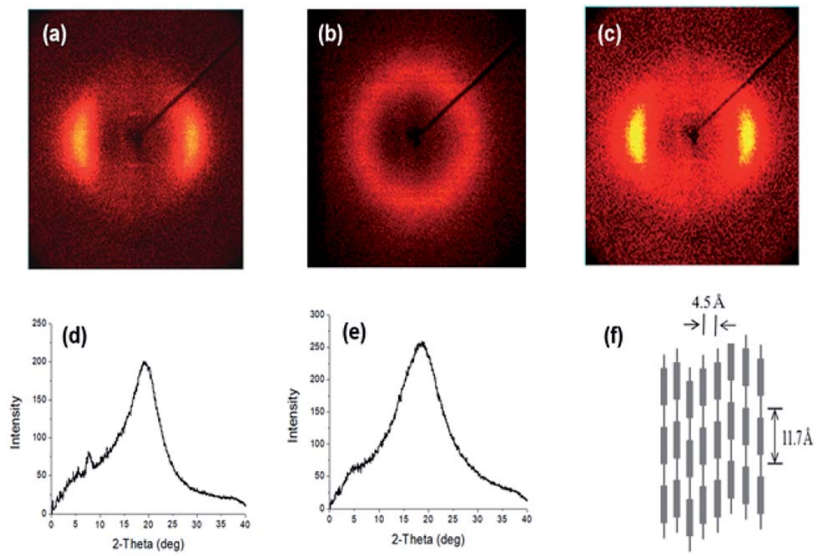

Fig. 3 XRD patterns of (a) PDEN1 at $170{ }^{\circ} \mathrm{C}$, (b) PDEN1 at $230{ }^{\circ} \mathrm{C}$, (c) PDEN1R at $170{ }^{\circ} \mathrm{C}$, (d) PDEN1 at $170{ }^{\circ} \mathrm{C}$, (e) PDEN1 at $230{ }^{\circ} \mathrm{C}$, (f) schematic of PDEN1 LC ordering. can also be seen at a diffraction angle of $2 \theta=19-20^{\circ}$, which corresponds to equatorial scans of 4.5-4.7 A. Since we used the same mesogenic units (LCE1 and LCE3) as before, their diffraction patterns are quite very similar. ${ }^{19}$ LC chains typically produce diffraction peaks corresponding to a lateral-packing of 3-6 А., ${ }^{\mathbf{1 , 2 3 , 2 4}}$ The peaks in the wide angle region are broader than those of PDEA1 and PDEA3, which indicates that the ordering of the PDEN1 and PDEN3 chains is more liquid-like (less ordered) due to their bulkier side groups. As the temperature rises to $230{ }^{\circ} \mathrm{C}$, the small angle reflections disappear while the broad peak at wide angles remains intact, which means that there is a breakdown in chain regularity and in the nematic phase output at that temperature. ${ }^{25}$

The thermal behaviors of the polymers were investigated by using the DSC and TGA. A single endothermic melting point appears in each of the DSC thermograms during the heating scans (Fig. 4(a)). By recording second heating thermograms to remove the complex thermal histories of the samples, we were able to determine their glass transition temperatures $\left(T_{\mathrm{g}}\right)$, and melting temperatures $\left(T_{\mathrm{m}}\right)$, which are listed in Table 1 . Above $T_{\mathrm{g}}$, chain relaxation and flexibility become evident. Increasing the length of the flexible spacer in the main chain reduces the rigidity of the polymer backbone by decreasing the proportion of the mesogen unit. ${ }^{19}$ Thus PDEN3 has a lower $T_{\mathrm{g}}$ than PDEN1 because of its soft backbone, but has a higher $T_{\mathrm{m}}$ due to the greater ordering of its alkyl groups. Both polymers, however, have a low melting temperature $\left(175^{\circ} \mathrm{C}\right.$ for PDEN1 and $194{ }^{\circ} \mathrm{C}$ for PDEN3), so they can easily be processed in an extruder or injection molding machine. Table 1 also provides the degradation temperatures ( $T_{\mathrm{dg}}$ of $5 \mathrm{wt} \%$ loss) of the polymers. The TGA thermograms in Fig. 4(b) show that all polymers have high thermal stabilities. Their degradations starts at temperatures above $350{ }^{\circ} \mathrm{C}$ and proceeds in a single stage, which indicates that the presence of the flexible spacer groups does not affect the high thermal stability of the lengthy cores with ester linkers.

The optical textures of the polymers near the transition temperature are presented in Fig. 5. Both polymers exhibit the birefringence characteristics of nematic liquid crystalline mesophase textures (the marbled nematic mesophase) near the transition temperature. ${ }^{26}$ Since these images were collected during the second heating process, they have characteristic birefringent textures even at temperatures lower than the transition temperature. The colorful textures remain almost unchanged when the samples are cooled to $25{ }^{\circ} \mathrm{C}$ after the first heating, which means that the mesophases of the polymers formed at high temperatures are stable. Fibril formation was
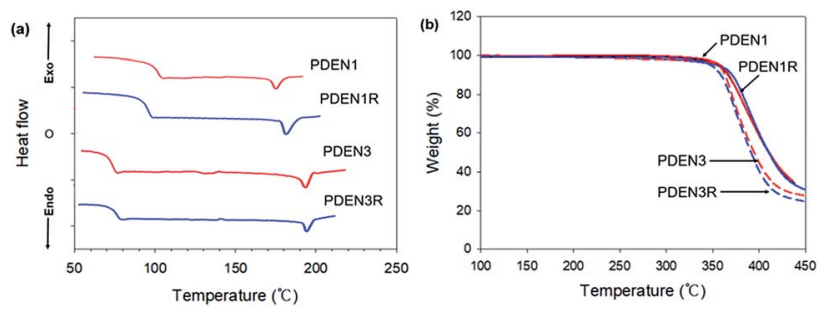

Fig. 4 (a) DSC thermograms and (b) TGA degradation curves. 


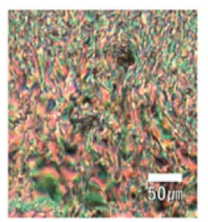

PDEN1 at $170^{\circ} \mathrm{C}$

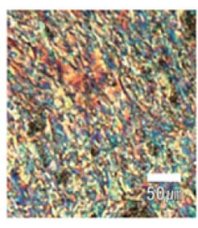

PDEN1R at $170^{\circ} \mathrm{C}$

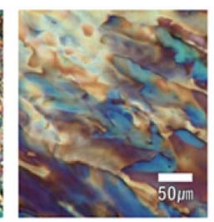

PDEN3 at $190^{\circ} \mathrm{C}$

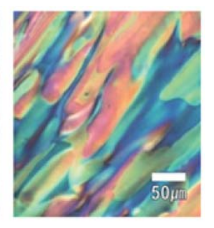

PDEN3R at $190^{\circ} \mathrm{C}$
Fig. 5 POM images of PDEN1, PDEN1R, PDEN3, and PDEN3R respectively.

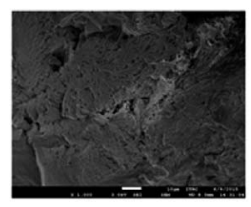

PDEN1 (normal)

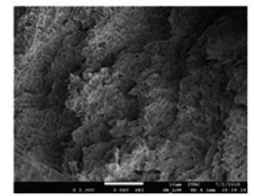

PDEN3 (normal)

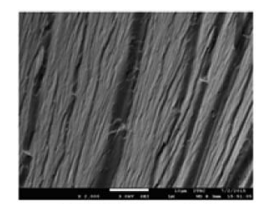

PDEN1 (parallel)

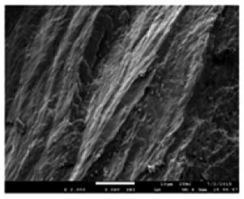

PDEN3 (parallel)

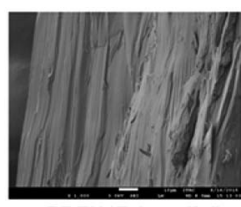

PDEN1R (parallel)

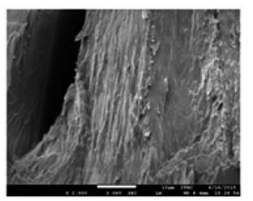

PDEN3R (parallel)
Fig. 6 SEM micrographs of fractured polymer fibrils (upper row: PDEN1 fractured surface normal to fibril direction (left); parallel to fibril direction (center); PDEN1R fractured surface parallel to fibril direction (right); lower row: PDEN3 fractured surface normal to fibril direction (left); parallel to fibril direction (center); PDEN3R fractured surface parallel to fibril direction (right)) (×2000 magnification).

confirmed with SEM, by examining the surface morphologies of the melt-drawn fibers (Fig. 6). All the samples contain welloriented TLCP fibrils. Protruding microfibrils can be seen in the cross-sections of the fibers after cryogenic fracturing in liquid nitrogen. These fibrils protrude above the fractured surfaces, and are thus part of continuous fibril morphologies on the TLCP surfaces that are oriented parallel to the fibril direction. ${ }^{13,19,27}$

\section{Conclusions}

New liquid crystalline polymers with low melting temperatures have been successfully synthesized by performing polyaddition reactions between diepoxide molecules and a primary monoamine (1-naphthylamine). Although the polymers synthesized via the reactive extrusion process have lower molar masses than the same polymers prepared in bulk reactions due to their short reaction time in the extruder, this approach is commercially very significant because of its potential for the mass production. The polymers synthesized with bulk polymerizations have high molar masses, which means that it is possible to produce high molar mass TLCPs in a commercial extruder that provides sufficient residence time for the reactants.

The polymers have good thermal stabilities and high decomposition temperatures above $350{ }^{\circ} \mathrm{C}$, which means that they can withstand high temperature applications. They also have low melting temperatures, which enables easy processing. PDEN3 has longer spacer groups and thus a high transition temperatures, but the thermal stabilities of the two polymers (PDEN1 and PDEN3) are comparable because the spacer groups are not so long (methyl and propyl). The polymers synthesized with the reactive polymerization process have nearly the same thermal properties as those synthesized with bulk polymerization. All the polymers exhibit a nematic liquid crystal mesophase morphology in the melt state. The polymers were found to form well-ordered structures upon cooling. After drawing, the stretched polymer fibers exhibit strong molecular orientation on the surface along the direction of flow. The many microfibrillar structures that protrude out perpendicular to the direction of flow from the fractured surface mean that these nematic polymers can be used in the preparation of in situ composites.

\section{Conflicts of interest}

The authors declare that there are no conflicts of interest.

\section{Acknowledgements}

This work was supported by KNRF (MSE BK21 Program of SNU) and MIKE (No. 10067685, Core Original Materials Development Program) and the Institute of Engineering Research at Seoul National University for the DSC measurements.

\section{Notes and references}

1 C. Tschierske, Angew. Chem., Int. Ed., 2013, 52, 8828-8878.

2 Thermotropic Liquid Crystal Polymers: Thin Polymerization, Characterization, Blends, and Applications, ed. T. S. Chung, 2001, Technomic Publishing Co. Inc., Lancaster, PA.

3 Liquid Crystalline Polymers: Vol 1-Structure and Chemistry, ed. V. K. Tahkur and M. R. Kessler, 2016.

4 A. M. Donald, A. H. Windle and S. Hanna, Liquid Crystalline Polymers (Cambridge Solid State Science), Cambridge Univ. Press, Cambridge, 2nd edn, 2006.

5 E. Burrell II and J. F. Johnson, J. Macromol. Sci. Rev. Macromol., 1979, 7, 137-170.

6 B. A. San Jose and K. Akagi, Polym. Chem., 2014, 5, 37373749.

7 M. Sato, Y. Kobayashi, T. Shimizu and I. Yamaguchi, Polym. Chem., 2010, 1, 891-898.

8 S. Zhang, C. Zhang, J. Wang, F. Hong, X. Hao, A. Zhang, Y. Wang, H. Wu, W. Zhang and J. Pua, Polym. Chem., 2016, 7, 3013-3025.

9 Y. Seo, S. M. Hong and K. U. Kim, Macromolecules, 1997, 30, 2978-2988.

10 Y. Seo, T. H. Ninh, S. M. Hong, S. Kim, T. J. Kang, H. S. Kim and J. Kim, Langmuir, 2006, 8, 3062-3067.

11 E. K. Fleischmann and R. Zentel, Angew. Chem., Int. Ed., 2013, 52, 8810-8827.

12 Y. Seo, B. Kim, S. Kwak and K. U. Kim, Polymer, 1999, 40, 4483-4492.

13 Y. Seo, B. Kim and K. U. Kim, Polymer, 1999, 40, 4441-4450. 14 Y. Seo, S. M. Hong, S. S. Hwang, T. S. Park, K. U. Kim, S. M. Lee and J. Lee, Polymer, 1995, 36, 515-524. 
15 Y. Seo, S. M. Hong, S. S. Hwang, T. S. Park, K. U. Kim, S. M. Lee and J. Lee, Polymer, 1995, 36, 525-534.

16 S. M. Lee, S. M. Hong, Y. Seo, T. S. Park, S. S. Hwang, K. U. Kim and J. Lee, Polymer, 1994, 35, 519-531.

17 Thermotropic Liquid Crystal Polymer Blends, ed. F. R. La Mantia, 1993, Technomic Publishing, Lancaster, PA.

18 R. K. Krishwaswamy and D. G. Baird, Polymer, 1999, 40, 701716.

19 K. Oh, H. Kim and Y. Seo, RSC Adv., 2017, 7, 29772-29778.

20 B. Mossey-Lesczak and M. W. Locharska, in Liquid Crystalline Compounds and Polymers as Materials of the XXI Century from Syntheses to Applications, ed. A. Juan and E. Schab-Balerzak, Trans-World Research Network, Kerala, India, 2011, pp.125-152.
21 N. S. Enikolopiyan, Pure Appl. Chem., 1976, 48, 317-328.

22 M. Blanco, M. A. Corcuera, C. C. Riccard and I. Mandryar, Polymer, 2005, 46, 7989-8000.

23 J. Watanabe and M. Hayashi, Macromolecules, 1989, 22, 4083-4088.

24 S. W. Kantor, T. C. Sung and E. D. T. Atkins, Macromolecules, 1992, 25, 2789-2795.

25 Z. Zhu, J. Zhi, A. Liu, H. Tang, W. Qiao, X. Wan and Q. Zhou, J. Polym. Sci., Part A-1: Polym. Chem., 2007, 45, 800-847.

26 C. Ryu, Y. Seo, Y. S. S. Hwang, S. M. Hong, T. S. Park and K. U. Kim, Int. Polym. Process., 1994, 9, 266-272.

27 B. Chena, S. Tsaya and J. Chen, Polymer, 2005, 46, 86248633. 